\title{
Adjuvant chemotherapy in gastric cancer
}

Keywords: gastric carcinoma, cancer, treatment selection, histological subtypes, treatment guidelines

\section{Introduction}

Gastric carcinoma (GC) is one of the most frequent cancer types worldwide. The GC is known to be the $5^{\text {th }}$ most common cancer type; however, the frequency of cancer differs across the regions of the world. In Japan, GC is one of the most common cancer types, while it is not common cancer. ${ }^{1,2}$ Although GC incidence decreasing steadily since the beginning of the $20^{\text {th }}$ century, it is still one major cause of cancer-related death in the United States. The mortality rate is strictly in relationship with the stage at the time of presentation. The 5-year survival rates drop from $70-75 \%$ to $35 \%$ when the patients compared less than stage IIB and beyond. ${ }^{3}$ In patients with early GC (T1N0) endoscopic or surgical resection is curative nearly $90 \%$ of the patients. The survival rates significantly drop in patients with T2-3 or regional lymph node-positive groups, especially in Caucasian ethnicity. ${ }^{4}$ Interestingly, in the T2-3 group, the prognosis is better in Asian patients when compared non-Asian population. ${ }^{5}$ Different histological subtypes determined in GC, which have prognostic information. Diffuse GC is reported to have a worse prognosis than the intestinal type. Although several molecular subtypes described in $\mathrm{GC}$, these subtypes do not affect the treatment selection. ${ }^{6,7}$

The current treatment guidelines of GC differ across the world. Perioperative chemotherapy is the standard procedure in Europe in clinically stage II-III disease, while post-operative chemotherapy is suggested in pathologically proven stage II-III GC. Although these guidelines offer different treatment modalities, both of them agree on D2 lymph node dissection, experienced surgeons and high-volume centers. ${ }^{8-10}$ The treatment of the early stage GC should include a multidisciplinary approach; however, the surgical resection and the lymph node dissection are the cornerstones of the treatment. There are different therapeutic options in Asia and the USA. In Asia, D2 dissection is routinely performed while in the USA, D1 dissection is still a mostly selected option. ${ }^{10,11}$ Although, post-operative mortality in $\mathrm{D} 2$ dissection is reported to be higher compared to D1 dissection, disease-specific mortality is lesser in D2 dissection. ${ }^{12}$

\section{Adjuvant chemotherapy trials}

One of the most effective studies in the adjuvant treatment of GC is MAGIC (United Kingdom Medical Research Council Adjuvant Gastric Infusional Chemotherapy) trial. Five hundred and three patients consisting of GC, distal esophagus and esophagogastric junction tumors (EGJ) were enrolled in the study. The patients randomly assigned to the surgery \pm epirubicin, cisplatin and infusional fluorouracil arms. In chemotherapy, arm patients received six cycles of chemotherapy, which was divided equally pre and postoperatively. The included patients were clinically $\mathrm{T} 2$ or higher, locally advanced and no evidence of distant metastasis. In chemotherapy arm, more patients had curative surgery and achieved post-operative down staging. Chemotherapy was well tolerated, receiving patients had $23 \%$ of neutropenia and $12 \%$ of grade $3-4$ adverse events. Although chemotherapy was well tolerated, only $42 \%$ of the patients had completed chemotherapy protocol. The patients had a major problem in completing the post-operative chemotherapy cycles. Despite the
Volume II Issue 3 - 2020

Ismail Beypinar,' Ahmet Taner Sumbul'
'Department of Medical Oncology, Eskișehir City Hospital,
Turkey
${ }^{2}$ Department of Medical Oncology, Baskent University, Turkey

Correspondence: Ahmet Taner Sümbül,Associate Professor, Department of Medical Oncology, Faculty of Medicine, Baskent University, Adana kılsa saglikyerleskesi,Yuregir,Adana, Turkey, Zipcode: 0 I I 30, Tel +905056 I66338, Fax 90322344 4445, Email drtanersu@yahoo.com

Received: February 27, 2020 | Published: June 30, 2020

low rate of completing the study protocol in the chemotherapy arm, peri-operative chemotherapy was significantly prolonged disease-free survival (DFS) and overall survival (OS). ${ }^{13}$

The CLASSIC trial was designed to prove the benefit of the adjuvant treatment in GC. In this trial, patients enrolled in surgery (with $\mathrm{D} 2$ dissection) \pm capecitabine $\left(1000 \mathrm{mg} / \mathrm{m}^{2}\right.$ twice daily on days 1 to 14$)$ and oxaliplatin $\left(130 \mathrm{mg} / \mathrm{m}^{2}\right.$ on day 1$)$. Two of three patients were received the pre-planned chemotherapy protocol. Ninety percent of the patients needed dose modifications due to adverse events. Despite lack of optimal treatment in the chemotherapy arm, a significant DFS (74 versus 59 percent, HR for death $0.56,95 \%$ CI $0.44-0.72$ ) and a borderline OS (83 versus 78 percent, HR $0.72,95 \%$ CI 0.52-1.00) benefits were achieved. However longer follow-up had resulted with a significant OS benefit in 5 years ( 78 versus 69 percent, HR for death $0.66,95 \%$ CI $0.51-0.85) .{ }^{14}$

In FLOT4-AIO trial, FLOT regimen (docetaxel $50 \mathrm{mg}$ / $\mathrm{m}^{2}$, oxaliplatin $85 \mathrm{mg} / \mathrm{m}^{2}$, and LV $200 \mathrm{mg} / \mathrm{m}^{2}$ with short-term infusional FU $2600 \mathrm{mg} / \mathrm{m}^{2}$ as a 24-hour infusion, all on day one and administered every two weeks) compared with ECF or ECX regimen. In a phase II part of the trial, which was composed of EGJ tumors, FLOT regimen was associated with higher rates of complete pathological response. Although both arms had a perioperative complication during the healing period, the FLOT regimen had significantly less adverse events when compared ECF/ECX regimen. In the phase III component of the trial, the rates of completing pre-planned post-surgery protocol were significantly higher in the FLOT group. Adverse events were higher in the ECF/ECX group. At a median follow-up of 43 months, FLOT was associated with a significantly greater median overall survival (50 versus 35 months, HR $0.77,95 \%$ CI $0.63-0.94)$ and three-year overall survival (57 versus 48 percent). ${ }^{15}$

The ACTS-GC trial was performed in Japan which was investigating the benefit of the S1 (a prodrug of fluorouracil not available in the US and Europe). The patients have enrolled in surgery \pm one year of S1 treatment. All patients underwent at least D2 dissection which has resulted in low local recurrence rates surgery only arm (2.8\%). S1 treatment improved both DFS and OS when compared with surgery. This trial showed even the highest quality of surgery was not sufficient to prevent the relapse. ${ }^{16}$

An EORTC and European ICCG study, which was underpowered to show $10 \%$ of the difference between groups, compared adjuvant 
5-fluorouracil + adriamycin (FAMTX) or epirubicin and methotrexate with leucovorin rescue (FEMTX) with surgery. The compared arms had no difference in terms of OS and DFS. Also, the dissection type of surgery was not certain. ${ }^{17}$

In a trial which recruited patients in Asia, adjuvant S-1 and docetaxel compared. The results of the JACCROGC-07 trial were announced in ASCO 2018. The 3-year DFS was favored S1 when compared to docetaxel (65.9\% versus $49.6 \%$ ) (HR $0.632,99 \%$ CI $0.400 \sim 0.998, \mathrm{p}=0.0007){ }^{18}$

In a French trial named FNCLCC/FFCD surgery \pm cisplatin $\left(100 \mathrm{mg} / \mathrm{m}^{2}\right.$ on day 1$)$ and infusional fluorouracil $\left(800 \mathrm{mg} / \mathrm{m}^{2} 1-5\right.$ days of 28) were compared. Although the rates of completing peri-operative protocol were low (47.8\%) like MAGIC trial, 5-year survival rates were higher in the chemotherapy group (38\% vs. $24 \%){ }^{19}$

\section{Conclusion}

Multiple trials evaluated peri-operative treatment in GC. The current guidelines offer different modalities due to possible different geographical, genetic, and technical features of the populations. Although peri-operative treatment strongly recommended for locally advanced GC patients, attention must be exercised for comorbidities and performance status. Also, adjuvant treatment had an OS benefit especially in high-risk patients. Post-operative treatment is appropriate for high-risk patients who had up-front surgery.

\section{Acknowledgments}

None.

\section{Conflicts of interest}

The authors declare there are no conflicts of interest.

\section{Funding}

None.

\section{References}

1. Iarc. IA for R on CWHO. GLOBOCAN 2012: Estimated Cancer Incidence, Mortality and Prevalence Worldwide in 2012. Globocan. 2012.

2. Torre LA, Bray F, Siegel RL, et al. Global cancer statistics, 2012. $C A$ Cancer J Clin. 2015;65(2):87-108.

3. Siegel RL, Miller KD, Jemal A. Cancer statistics, 2019. CA Cancer J Clin. 2019;69(1):7-34.

4. Reim D, Loos M, Vogl F, et al. Prognostic implications of the seventh edition of the international union against cancer classification for patients with gastric cancer: The western experience of patients treated in a single-center European institution. J Clin Oncol. 2013;31(2):263271 .

5. Lin SJ, Gagnon-Bartsch JA, Tan IB, et al. Signatures of tumour immunity distinguish Asian and non-Asian gastric adenocarcinomas. Gut. 2015;64(11):1721-1731.
6. Cristescu R, Lee J, Nebozhyn M, et al. Molecular analysis of gastric cancer identifies subtypes associated with distinct clinical outcomes. Nat Med. 2015;21(5):449-456.

7. Bass AJ, Thorsson V, Shmulevich I, et al. Comprehensive molecular characterization of gastric adenocarcinoma. Nature. 2014;513(7517):202-209.

8. Smyth EC, Verheij M, Allum W, et al. Gastric cancer: ESMO clinical practice guidelines for diagnosis, treatment and follow-up. Ann Oncol. 2016;27(suppl 5):v38-v49.

9. Lutz MP, Zalcberg JR, Ducreux M, et al. Highlights of the EORTC st. gallen international expert consensus on the primary therapy of gastric, gastroesophageal and oesophageal cancer - Differential treatment strategies for subtypes of early gastroesophageal cancer. Eur J Cancer. 2012;48(16):2941-2953.

10. Japanese Gastric Cancer Association. Japanese gastric cancer treatment guidelines 2014 (ver. 4). Gastric Cancer. 2017;20(1):1-19.

11. Ajani JA, D’Amico TA, Almhanna K, et al. Gastric Cancer, Version 3.2016; Clinical Practice Guidelines in Oncology. J Natl Compr Cancer Netw. 2016;14(10):1286-1312.

12. Mocellin S, Mcculloch P, Kazi H, et al. Extent of lymph node dissection for adenocarcinoma of the stomach. Cochrane Database Syst Rev. 2015;2015(8):CD001964

13. Cunningham D, Allum WH, Stenning SP, et al. Perioperative Chemotherapy versus Surgery Alone for Resectable Gastroesophageal Cancer. N Engl J Med. 2006;355(1):11-20.

14. Noh SH, Park SR, Yang HK, et al. Adjuvant capecitabine plus oxaliplatin for gastric cancer after D2 gastrectomy (CLASSIC): 5-year follow-up of an open-label, randomised phase 3 trial. Lancet Oncol. 2014;15(12):1389-1396.

15. Al-Batran SE, Homann N, Pauligk C, et al. Perioperative chemotherapy with fluorouracil plus leucovorin, oxaliplatin, and docetaxel versus fluorouracil or capecitabine plus cisplatin and epirubicin for locally advanced, resectable gastric or gastro-oesophageal junction adenocarcinoma (FLOT4): a randomised, phase 2/3 trial. Lancet. 2019;393(10184):1948-1957.

16. Sakuramoto S, Sasako M, Yamaguchi T, et al. Adjuvant chemotherapy for gastric cancer with S-1, an oral fluoropyrimidine. $N$ Engl J Med. 2007;357(18):1810-1820.

17. Nitti D, Wils J, Dos Santos JG, et al. Randomized phase III trials of adjuvant FAMTX or FEMTX compared with surgery alone in resected gastric cancer. A combined analysis of the EORTC GI Group and the ICCG. Ann Oncol. 2006;17(2):262-269.

18. Kodera Y, Yoshida K, Kochi M, et al. A randomized phase III study comparing S-1 plus docetaxel with $\mathrm{S}-1$ alone as a postoperative adjuvant chemotherapy for curatively resected stage III gastric cancer (JACCRO GC-07 trial). J Clin Oncol. 2018;36(15).

19. Ychou M, Boige V, Pignon JP, et al. Perioperative chemotherapy compared with surgery alone for resectable gastroesophageal adenocarcinoma: an FNCLCC and FFCD multicenter phase III trial. $J$ Clin Oncol. 2011;29(13):1715-1721. 